\begin{tabular}{lll}
\hline VOL. 80 & 1999 & NO. 2 \\
\hline
\end{tabular}

\title{
A PALEY-WIENER THEOREM ON NA HARMONIC SPACES
}

BY

FRANCESCA ASTENGO AND BIANCA DI BLASIO (TORINO)

\begin{abstract}
Let $N$ be an $H$-type group and consider its one-dimensional solvable extension $N A$, equipped with a suitable left-invariant Riemannian metric. We prove a PaleyWiener theorem for nonradial functions on $N A$ supported in a set whose boundary is a horocycle of the form $N a, a \in A$.
\end{abstract}

1. Introduction. A classical problem in harmonic analysis is to characterize the image under the Fourier transform of functions with given support.

The theorems concerning this subject are usually called Paley-Wiener type theorems. Among these we recall the following classical result for the real Fourier transform $\mathcal{F}$ in $\mathbb{R}^{n}$.

TheOREm A. Let $f$ be a function in the Schwartz space $\mathcal{S}\left(\mathbb{R}^{n}\right)$ and let $a \in \mathbb{R}$. The support of $f$ is contained in the set $\left\{\left(x_{1}, \ldots, x_{n}\right) \in \mathbb{R}^{n}: x_{n} \geq a\right\}$ if and only if for every $\xi^{\prime}$ in $\mathbb{R}^{n-1}$ the function $\xi_{n} \mapsto \mathcal{F} f\left(\xi^{\prime}, \xi_{n}\right)$ extends to a holomorphic function in $\operatorname{Im}_{-}=\left\{\xi_{n} \in \mathbb{C}: \operatorname{Im} \xi_{n}<0\right\}$ such that

$$
\sup _{\left(\xi^{\prime}, \xi_{n}\right) \in \mathbb{R}^{n-1} \times \overline{\operatorname{Im}_{-}}}\left|\mathcal{F} f\left(\xi^{\prime}, \xi_{n}\right)\right| e^{-a \operatorname{Im} \xi_{n}}\left(1+\left|\xi^{\prime}\right|+\left|\xi_{n}\right|\right)^{l}<\infty \quad \forall l \in \mathbb{N} .
$$

This theorem can be extended to the class of Schwartz functions whose support is contained in a set with any hyperplane as boundary. An analogous result was obtained by J. Faraut $[F]$ in the case of noncompact rank one symmetric spaces.

The purpose of this paper is to find an analogue of Theorem A in the case of the solvable $N A$ groups introduced by E. Damek [Da2]. Such a group is a one-dimensional extension of a two-step nilpotent Lie group $N$ of Heisenberg type [Ka1], obtained by letting $A=\mathbb{R}^{+}$act on $N$ by anisotropic dilations. One can endow $N A$ with a suitable left-invariant Riemannian metric which makes it a harmonic manifold [DaR2]. This class of $N A$ groups includes all noncompact symmetric spaces $G / K$ of rank one, where $G=N A K$ is the Iwasawa decomposition of the connected simply connected semisimple Lie group $G$ of rank one with finite center and $K$ is a maximal compact subgroup

1991 Mathematics Subject Classification: Primary 43A30; Secondary 43A80, 22E25. 
of $G$ (the real hyperbolic spaces fit into this framework as degenerate cases). There are $N A$ groups such that the center of the Lie algebra of $N$ is of any given dimension [Ka1], hence most of them are nonsymmetric spaces.

We denote by $\mathcal{S}(N A)$ the space of Schwartz functions on the group $N A$ (see Section 4 for the definition and $[\mathrm{GV}, \mathrm{V}]$ for the symmetric case). Let $f$ be in $\mathcal{S}(N A)$ and denote by $\widehat{f}$ its Helgason-Fourier transform, defined as in $[\mathrm{ACD}]$ by

$$
\widehat{f}(\lambda, n)=\int_{N A} f(x) \mathcal{P}_{\lambda}(x, n) d x \quad \forall \lambda \in \mathbb{R}, \forall n \in N,
$$

where $\mathcal{P}_{\lambda}$ is a complex power of the Poisson kernel (see Section 3, and see $[\mathrm{He}]$ for the symmetric case).

In the context of nonsymmetric harmonic spaces we prove

TheOREM B. Let $f$ be in $\mathcal{S}(N A)$ and $\tau$ be a real number. The support of $f$ is contained in the set $E_{\tau}=\left\{n a \in N A: a \geq e^{\tau}\right\}$ if and only if the following conditions hold:

(i) $\lambda \mapsto \widehat{f}(\lambda, n)$ is holomorphic in $\{\lambda \in \mathbb{C}: \operatorname{Im} \lambda>0\}$;

(ii) $(\lambda, n) \mapsto \widehat{f}(\lambda, n)$ is $C^{\infty}(\{\lambda \in \mathbb{C}: \operatorname{Im} \lambda \geq 0\} \times N)$;

(iii) for every positive integer $l$ and for $1<p \leq \infty$,

$$
\sup _{\operatorname{Im} \lambda \geq 0}\|\widehat{f}(\lambda, \cdot)\|_{L^{p}(N)}(1+|\lambda|)^{l} e^{\tau \operatorname{Im} \lambda}<\infty .
$$

In Section 7 we shall see that it is sufficient to verify condition (iii) for a certain $p$ to prove that $f$ is supported in $E_{\tau}$. Moreover, notice that the boundary of $E_{\tau}$ is the orbit $N e^{\tau}$, which is easily seen to be a horocycle, a generalization of a hyperplane in $\mathbb{R}^{n}$.

The necessity part of the proof of Theorem B is fairly easy and follows from the formula for the Poisson kernel. The sufficiency part is nontrivial and uses the Gelfand transform of the commutative algebra $L^{1}(N)^{\natural}$ of biradial integrable functions on $N$. Therefore in Section 5 we describe the Gelfand spectrum of $L^{1}(N)^{\natural}$. The section generalizes to non-Iwasawa $N$ groups the analysis of $M$-invariant functions on $N$ for the symmetric case ( $M$ is the centralizer of $A$ in $K$ ). Due to the lack of the group $K$ (see [Da3]), we exploit the theory of averaging projectors developed by Damek and F. Ricci [DaR1]. We need a new averaging projector on the group $N$ to analyze biradial functions.

The proof of Theorem B is inspired by that given by Faraut $[\mathrm{F}]$ for an analogous theorem in the context of rank one symmetric spaces. Instead of condition (iii) in Theorem B, Faraut has 
(iii)' for every positive integer $l$,

$$
\sup _{\operatorname{Im} \lambda \geq 0}\left\|\Delta_{j} \widehat{f}(\lambda, \cdot)\right\|_{L^{1}(N)}(1+|\lambda|)^{l} e^{\tau \operatorname{Im} \lambda}<\infty, \quad j=1,2,
$$

where $\Delta_{1}$ and $\Delta_{2}$ are suitable sublaplacians on $N$ (see Section 5 for the definition).

The technique used in Faraut's and in our paper (with minor modifications) can be applied to extend Faraut's result to all $N A$ harmonic spaces. However, Faraut uses the theory of Whittaker vectors, which we can avoid with the help of the material of Section 5 and some results derived from $[\mathrm{CH}]$.

Other results concerning Paley-Wiener type theorems on $N A$ harmonic spaces can be found in [ADY, Di, Ri2] for the radial case. For nonradial $C^{\infty}$ functions with compact support in the symmetric case see [He]; in the nonsymmetric case a partial result has been obtained in [ACD], but it seems that the full characterization is nontrivial to prove.

Our paper is organized as follows: Section 2 contains some notation and background material, and recalls the main facts used in the sequel; Section 3 deals with the Poisson kernel and the Helgason-Fourier transform. In Section 4 we prove the necessity part of Theorem B. In Section 5 we determine the Gelfand spectrum of $L^{1}(N)^{\natural}$. In Section 6 we evaluate the Gelfand transforms of the powers of the Poisson kernel and we find their asymptotic expansions. Finally, in Section 7, we complete the proof of Theorem B, demonstrating the sufficiency of our conditions.

The authors would like to thank Jean-Philippe Anker and Fulvio Ricci for their suggestions and comments.

2. Preliminaries. We have divided this section into two subsections to make it more readable. The first subsection deals with groups $N$ of Heisenberg type and their representations; the second one with harmonic extensions $N A$ of Heisenberg type groups.

2.1. Groups of Heisenberg type. Let $\mathbf{n}$ be a two-step real nilpotent Lie algebra endowed with an inner product $\langle,\rangle_{\mathbf{n}}$. Write $\mathbf{n}$ as an orthogonal sum $\mathbf{n}=\mathbf{v} \oplus \mathbf{z}$, where $\mathbf{z}=[\mathbf{n}, \mathbf{n}]$ is the center of $\mathbf{n}$.

For each $Z$ in $\mathbf{z}$, define the map $J_{Z}: \mathbf{v} \rightarrow \mathbf{v}$ by

$$
\left\langle J_{Z} X, Y\right\rangle_{\mathbf{n}}=\langle[X, Y], Z\rangle_{\mathbf{n}} \quad \forall X, Y \in \mathbf{v} .
$$

Definition [Ka1]. The Lie algebra $\mathbf{n}$ is called an H-type algebra if, for every $Z$ in $\mathbf{z}$,

$$
J_{Z}^{2}=-|Z|^{2} I_{\mathbf{v}},
$$


where $I_{\mathbf{v}}$ is the identity on $\mathbf{v}$. A connected and simply connected Lie group $N$ is called an $H$-type group if its Lie algebra is an $H$-type algebra.

Note that for every unit $Z$ in $\mathbf{z}$, the map $J_{Z}$ defines a complex structure on $\mathbf{v}$, so that $\mathbf{v}$ has even dimension $2 m$.

Since $\mathbf{n}$ is a nilpotent Lie algebra, the exponential map is surjective. We can then parametrize the elements of $N=\exp \mathbf{n}$ by $(X, Z)$, for $X$ in $\mathbf{v}$ and $Z$ in z. By the Campbell-Hausdorff formula it follows that the product law in $N$ is

$$
(X, Z)\left(X^{\prime}, Z^{\prime}\right)=\left(X+X^{\prime}, Z+Z^{\prime}+\frac{1}{2}\left[X, X^{\prime}\right]\right) \quad \forall X, X^{\prime} \in \mathbf{v}, \forall Z, Z^{\prime} \in \mathbf{z} .
$$

We denote by $d X$ and $d Z$ the Lebesgue measure on $\mathbf{v}$ and on $\mathbf{z}$ respectively; it is easy to check that $d n=d X d Z$ is a Haar measure on $N$.

The unitary irreducible representations of $N$ fall into two classes: the first are trivial on the center and do not appear in the Plancherel formula; the others are parametrized by $\mathbb{R}^{+} \times S_{\mathbf{z}}$ (see [CH, Ri1]), where $S_{\mathbf{z}}=\{\omega \in$ $\mathbf{z}:|\omega|=1\}$ is the unit sphere in $\mathbf{z}$.

For $\omega$ in $S_{\mathbf{z}}$, we consider $\mathbf{v}$ endowed with the complex structure $J_{\omega}$. We denote by $I_{\omega}: \mathbf{v} \rightarrow \mathbb{C}^{m}$ the corresponding isomorphism and by $\{\cdot, \cdot\}_{\omega}$ the corresponding Hermitian inner product given by

$$
\{X, Y\}_{\omega}=\langle X, Y\rangle_{\mathbf{n}}+i\left\langle J_{\omega} X, Y\right\rangle_{\mathbf{n}} \quad \forall X, Y \in \mathbf{v} .
$$

We define $\mathcal{W}_{\nu, \omega}$ to be the space of functions $\xi: \mathbf{v} \rightarrow \mathbb{C}$ such that $\xi \circ I_{\omega}^{-1}$ : $\mathbb{C}^{m} \rightarrow \mathbb{C}$ is an entire function and

$$
\|\xi\|_{\nu}^{2}=\int_{\mathbf{v}}|\xi(X)|^{2} e^{-\nu|X|^{2} / 2} d X<\infty .
$$

Thus $\mathcal{W}_{\nu, \omega}$ is a Hilbert space with respect to the inner product associated with the norm \|\|$_{\nu}$. For any multiindex $j$ in $\mathbb{N}^{m}(\mathbb{N}=\{0,1,2, \ldots\})$ we denote by $\wp_{j, \nu}$ the following homogeneous normalized polynomial:

$$
\wp_{j, \nu}(X)=\pi^{-m / 2}(\nu / 2)^{(m+|j|) / 2}(j !)^{-1 / 2}\left(I_{\omega}(X)\right)^{j} \quad \forall X \in \mathbf{v},
$$

where $|j|=j_{1}+\ldots+j_{m}, j !=j_{1} ! \ldots j_{m}$ ! and $\zeta^{p}=\zeta_{1}^{j_{1}} \ldots \zeta_{m}^{j_{m}}$, for $\zeta$ in $\mathbb{C}^{m}$. One can check that the family $\left\{\wp_{j, \nu}\right\}_{j \in \mathbb{N}^{m}}$ is an orthonormal basis of $\mathcal{W}_{\nu, \omega}$.

For any $\nu$ in $\mathbb{R}^{+}$and any $\omega$ in $S_{\mathbf{z}}$ let $\pi_{\nu, \omega}$ be the unitary representation of $N$ on $\mathcal{W}_{\nu, \omega}$ defined, for every $(X, Z)$ in $N$, by

$$
\left[\pi_{\nu, \omega}(X, Z) \xi\right](Y)=e^{-\nu\left(|X|^{2} / 4+\{Y, X\}_{\omega} / 2+i\langle Z, \omega\rangle_{\mathbf{n}}\right)} \xi(X+Y) \quad \forall Y \in \mathbf{v} .
$$

As customary the representations $\pi_{\nu, \omega}$ can be viewed as representations of the Banach algebra $L^{1}(N)$ on $\mathcal{W}_{\nu, \omega}$ by setting

$$
\pi_{\nu, \omega}(f)=\int_{N} \pi_{\nu, \omega}(n) f(n) d n .
$$


The following inversion formula holds (see [CH, Ri1]):

$$
f(n)=\frac{\left|S_{\mathbf{z}}\right|}{(2 \pi)^{Q}} \int_{0}^{\infty} \int_{S_{\mathbf{z}}} \operatorname{tr}\left(\pi_{\nu, \omega}(f) \pi_{\nu, \omega}\left(n^{-1}\right)\right) \nu^{m+k-1} d s(\omega) d \nu
$$

for every $n$ in $N$, where $\left|S_{\mathbf{z}}\right|$ is the measure of the unit sphere $S_{\mathbf{z}}$ and $d s(\omega)$ is the normalized surface measure thereof.

2.2. Harmonic spaces. Let $N A$ be the semidirect product of the Lie groups $N$ and $A=\mathbb{R}^{+}$with respect to the action of $A$ on $N$ given by the dilations $(X, Z) \mapsto\left(a^{1 / 2} X, a Z\right)$. As customary we write $(X, Z, a)$ for the element $n a=\exp (X+Z) a$. It can easily be checked that the product law in $N A$ is given by

$$
(X, Z, a)\left(X^{\prime}, Z^{\prime}, a^{\prime}\right)=\left(X+a^{1 / 2} X^{\prime}, Z+a Z^{\prime}+\frac{1}{2} a^{1 / 2}\left[X, X^{\prime}\right], a a^{\prime}\right) .
$$

We denote by $k$ the dimension of the center $\mathbf{z}$, and by $Q=m+k$ the homogeneous dimension of $N$.

The left Haar measure on $N A$, unique up to a multiplicative constant, is given by

$$
d x=a^{-Q-1} d X d Z d a=a^{-Q-1} d n d a,
$$

where $d a$ is the Lebesgue measure on $\mathbb{R}^{+}$.

Note that the right Haar measure is $a^{-1} d X d Z d a$, hence the group $N A$ is not unimodular. This implies that the group $N A$ has exponential volume growth.

We endow $N A$ with the left-invariant Riemannian structure induced by the following inner product on the Lie algebra $\mathbf{n} \oplus \mathbb{R}$ of $N A$ :

$$
\left\langle(X, Z, \alpha),\left(X^{\prime}, Z^{\prime}, \alpha^{\prime}\right)\right\rangle=\left\langle X, X^{\prime}\right\rangle_{\mathbf{n}}+\left\langle Z, Z^{\prime}\right\rangle_{\mathbf{n}}+\alpha \alpha^{\prime},
$$

where $\alpha=\log a(a \in A)$. In [DaR2] it is proved that, as a Riemannian manifold, $N A$ is a harmonic space [RWW]. Rank one symmetric spaces of the noncompact type constitute a subclass of $N A$ harmonic spaces.

In $[\mathrm{CDKR}]$ it is proved that the geodesic distance of $x=(X, Z, a)$ from the identity $e$ of $N A$ is

$$
\varrho(x)=d(x, e)=\log \frac{1+r(x)}{1-r(x)},
$$

where $r(x)$ lies in the interval $(0,1)$ and is given by

$$
1-r(x)^{2}=\frac{4 a}{\left(1+a+|X|^{2} / 4\right)^{2}+|Z|^{2}} .
$$

Note that

$$
r(x)=\tanh \frac{\varrho(x)}{2} \quad \text { and } \quad 1-r(x)^{2}=\left(\cosh \frac{\varrho(x)}{2}\right)^{-2} .
$$


We fix an orthonormal basis $\left\{H, E_{1}, \ldots, E_{2 m}, U_{1}, \ldots, U_{k}\right\}$ adapted to the orthogonal decomposition of the Lie algebra of $N A$ as $\mathbb{R} \oplus \mathbf{v} \oplus \mathbf{z}$ and we write $X=\sum_{j=1}^{2 m} x_{j} E_{j}$ and $Z=\sum_{l=1}^{k} z_{l} U_{l}$ for $X$ in $\mathbf{v}$ and $Z$ in $\mathbf{z}$.

We keep the same notation for the left-invariant vector fields on the group $N$ corresponding to the vectors $E_{1}, \ldots, E_{2 m}, U_{1}, \ldots, U_{k}$. It is easy to check that for a smooth function $f$ on $N$ we have

$$
\begin{aligned}
& E_{j} f(X, Z)=\partial_{x_{j}} f(X, Z)+\frac{1}{2} \sum_{l=1}^{k}\left\langle J_{U_{l}} X, E_{j}\right\rangle_{\mathbf{n}} \partial_{z_{l}} f(X, Z) \\
& U_{l} f(X, Z)=\partial_{z_{l}} f(X, Z)
\end{aligned}
$$

for $j=1, \ldots, 2 m$ and $l=1, \ldots, k$.

The left-invariant vector fields extending to $N A$ the vectors $H, E_{1}, \ldots$ $\ldots, E_{2 m}, U_{1}, \ldots, U_{k}$ are respectively given by $a \partial_{a}, a^{1 / 2} E_{1}, \ldots, a^{1 / 2} E_{2 m}$, $a U_{1}, \ldots, a U_{k}$.

Moreover, Damek [Da1] has proved that the Laplace-Beltrami operator of the group $N A$ can be written as

$$
\mathcal{L}=a \sum_{j=1}^{2 m} E_{j}^{2}+a^{2} \sum_{l=1}^{k} U_{l}^{2}+\left(a \partial_{a}\right)^{2}-Q a \partial_{a} .
$$

3. The Poisson kernel and the Helgason-Fourier transform. For $n_{1}$ in $N$, define (see [Da1, CDKR]) the Poisson kernel on $N A$ at $n_{1}$ as the function

$$
\mathcal{P}\left(\cdot, n_{1}\right): N A \rightarrow \mathbb{R}, \quad n a \mapsto \mathcal{P}\left(n a, n_{1}\right)=P_{a}\left(n_{1}^{-1} n\right),
$$

where, for any $a>0, P_{a}(n)$ is the function on $N$ given by

$$
P_{a}(n)=P_{a}(X, Z)=a^{Q}\left(\left(a+\frac{|X|^{2}}{4}\right)^{2}+|Z|^{2}\right)^{-Q} .
$$

We use the following properties of the Poisson kernel:

$$
\begin{gathered}
\mathcal{L P}\left(\cdot, n_{1}\right)=0 \quad \forall n_{1} \in N, \\
P_{a}(n)=a^{-Q} P_{1}\left(a^{-1} n a\right) \quad \forall a \in A, \forall n \in N .
\end{gathered}
$$

It is easy to check that level sets of the Poisson kernel and sets of the form $\left\{n a \in N A: a=e^{\tau}\right\}$ are horocycles, that is, submanifolds orthogonal to all geodesics with the same endpoint. Horocycles can be viewed as a generalization of the hyperplanes in $\mathbb{R}^{n}$, which are submanifolds orthogonal to a fixed direction.

Define the kernel $\mathcal{P}_{\lambda}: N A \times N \rightarrow \mathbb{C}$ by

$$
\mathcal{P}_{\lambda}(x, n)=[\mathcal{P}(x, n)]^{1 / 2-i \lambda / Q} .
$$


In $[\mathrm{ACD}]$ the authors and R. Camporesi studied the Fourier analysis of smooth, compactly supported functions on $N A$. If $f$ is such a function, we defined its Fourier transform to be the function $\widehat{f}$ on $\mathbb{C} \times N$ given by the rule

$$
\widehat{f}(\lambda, n)=\int_{N A} f(x) \mathcal{P}_{\lambda}(x, n) d x \quad \forall \lambda \in \mathbb{C}, \forall n \in N ;
$$

we proved the following inversion formula for $f$ in $C_{\mathrm{c}}^{\infty}(N A)$ :

$$
f(x)=\frac{1}{4 \pi} \int_{\mathbb{R} \times N} \mathcal{P}_{-\lambda}(x, n) \widehat{f}(\lambda, n)|\mathbf{c}(\lambda)|^{-2} d \lambda d n \quad \forall x \in N A,
$$

where $\mathbf{c}(\lambda)$ is given by

$$
\mathbf{c}(\lambda)=\frac{2^{Q-2 i \lambda} \Gamma(2 i \lambda) \Gamma((2 m+k+1) / 2)}{\Gamma(Q / 2+i \lambda) \Gamma((m+1) / 2+i \lambda)} .
$$

4. The main theorem. If $U$ and $V$ belong to the universal enveloping algebra $\mathcal{U}$ of $N A$, denote respectively by $U^{\mathrm{L}} f$ and by $V^{\mathrm{R}} f$ the corresponding left-invariant and right-invariant vector fields applied to a $C^{\infty}$ function $f$ on $N A$. Often we shall simply write $U f$ for $U^{\mathrm{L}} f$.

Definition. Denote by $\mathcal{S}(N A)$ the space of $C^{\infty}$ functions $f$ on $N A$ such that

$$
\sup _{x \in N A} e^{Q \varrho(x) / 2}(1+\varrho(x))^{h}\left|\left(U^{\mathrm{L}} V^{\mathrm{R}} f\right)(x)\right|<\infty,
$$

for every positive integer $h$ and every $U, V \in \mathcal{U}$.

The Schwartz spaces for the symmetric case were first defined in $[\mathrm{HC}]$ and further studied in detail in $[\mathrm{GV}, \mathrm{V}]$. Note that the factor $e^{Q \varrho(x) / 2} \mathrm{com}-$ pensates the exponential volume growth of $N A$.

It can be verified that if $f$ is a function in $\mathcal{S}(N A)$ then its Fourier transform $\widehat{f}$ is well defined in $\{\lambda \in \mathbb{C}: \operatorname{Im} \lambda=0\} \times N$. Moreover, the inversion formula (3.2) holds for $f$ in $\mathcal{S}(N A)$.

We are interested in functions with support contained in sets of the type

$$
E_{\tau}=\left\{n a \in N A: a \geq e^{\tau}\right\}, \quad \tau \in \mathbb{R} .
$$

Note that the boundary of $E_{\tau}$ is a horocycle.

Definition. Let $1<p \leq \infty$. For every real number $\tau$ denote by $\mathcal{H}_{\tau, p}$ the space of functions $\psi$ defined on $\{\lambda \in \mathbb{C}: \operatorname{Im} \lambda \geq 0\} \times N$ such that

(i) $\lambda \mapsto \psi(\lambda, n)$ is holomorphic in $\{\lambda \in \mathbb{C}: \operatorname{Im} \lambda>0\}$;

(ii) $(\lambda, n) \mapsto \psi(\lambda, n)$ is $C^{\infty}(\{\lambda \in \mathbb{C}: \operatorname{Im} \lambda \geq 0\} \times N)$;

(iii) for every positive integer $h$ the following estimate holds:

$$
\sup _{\operatorname{Im} \lambda \geq 0}\|\psi(\lambda, \cdot)\|_{L^{p}(N)}(1+|\lambda|)^{h} e^{\tau \operatorname{Im} \lambda}<\infty .
$$


Theorem 4.1. Suppose that $\operatorname{dim} \mathbf{z}=k>1$. Let $f$ be in $\mathcal{S}(N A)$ and let $\tau$ be a real number. The following conditions are equivalent:

(1) the support of $f$ is contained in $E_{\tau}$;

(2) $\widehat{f}$ extends to a function belonging to $\mathcal{H}_{\tau, p}$ for every $p, 1<p \leq \infty$;

(3) $\widehat{f}$ extends to a function belonging to $\mathcal{H}_{\tau, p}$ for some $p, 1<p<$ $2 k /(k+1)$.

The proof that $(1) \Rightarrow(2)$ is contained in Proposition 4.3 below; $(2) \Rightarrow(3)$ is trivial, while the implication $(3) \Rightarrow(1)$ will be proved in Section 7 .

Our method does not work in the case $k=1$. This is due to the fact that the spherical functions in Proposition 5.3 are just in $L^{\infty}(N)$ and so the estimate (7.3) does not make sense. However, the following lemma and proposition cover also the case $k=1$.

LEMma 4.2. If $f$ is in $\mathcal{S}(N A)$ then, for every $U$ and $V$ in the universal enveloping algebra $\mathcal{U}$, there exists a constant $c>0$ such that

$$
\int_{N}\left|\left(U^{L} V^{R} f\right)(n a)\right| d n \leq c a^{Q / 2} .
$$

Proof. It is easy to check that

$$
\varrho\left(a^{1 / 2} n a^{1 / 2}\right) \geq \varrho(n) \quad \forall a \in A, \forall n \in N .
$$

Thus for $f$ in $\mathcal{S}(N A)$,

$$
\begin{aligned}
\int_{N}\left|\left(U^{L} V^{R} f\right)(n a)\right| d n & =\int_{N}\left|\left(U^{L} V^{R} f\right)\left(a^{1 / 2} n a^{-1 / 2} a\right)\right| a^{Q / 2} d n \\
& \leq c \int_{N} e^{-Q \varrho\left(a^{1 / 2} n a^{1 / 2}\right) / 2}\left(1+\varrho\left(a^{1 / 2} n a^{1 / 2}\right)\right)^{-h} a^{Q / 2} d n \\
& \leq c a^{Q / 2} \int_{N} e^{-(Q / 2) \varrho(n)}(1+\varrho(n))^{-h} d n \\
& =c a^{Q / 2},
\end{aligned}
$$

for a sufficiently large integer $h$.

Proposition 4.3. Let $f$ be in $\mathcal{S}(N A)$ and let $\tau$ be a real number. If the support of $f$ is contained in $E_{\tau}$, then $\widehat{f}$ extends to a function belonging to $\mathcal{H}_{\tau, p}$ for every $p, 1<p \leq \infty$.

Proof. We have to check that $\widehat{f}$ has properties (i), (ii) and (iii) in the definition of $\mathcal{H}_{\tau, p}$. We prove (i) by verifying that the integral

$$
\widehat{f}(\lambda, \bar{n})=\int_{N A} f(x) \mathcal{P}_{\lambda}(x, \bar{n}) d x
$$

converges uniformly for $(\lambda, \bar{n})$ in $\left\{\lambda \in \mathbb{C}: 0 \leq \operatorname{Im} \lambda \leq \lambda_{1}\right\} \times N$, for every $\lambda_{1}>0$, and applying the Morera theorem. Observe that for $0 \leq \operatorname{Im} \lambda \leq \lambda_{1}$ 
and $a>e^{\tau}$, by (3.1), we have

$$
\left|\mathcal{P}_{\lambda}(n a, \bar{n})\right|=\left|a^{-Q / 2+i \lambda} P_{1}\left(a^{-1} n^{-1} \bar{n} a\right)^{1 / 2-i \lambda / Q}\right| \leq C a^{-Q / 2},
$$

where $C=\max \left(1, e^{-\tau \lambda_{1}}\right)$. Let $f$ be in $\mathcal{S}(N A)$ with support contained in $E_{\tau}$; by Lemma 4.2 , we obtain

$$
\begin{aligned}
\int_{N A}\left|f(n a) \mathcal{P}_{\lambda}(n a, \bar{n})\right| a^{-Q-1} d n d a & \leq C \int_{N A}|f(n a)| a^{-(3 / 2) Q-1} d n d a \\
& \leq c \int_{e^{\tau}}^{\infty} a^{-Q-1} d a .
\end{aligned}
$$

Thus (4.1) converges uniformly in $\left\{\lambda \in \mathbb{C}: 0 \leq \operatorname{Im} \lambda \leq \lambda_{1}\right\} \times N$, for every $\lambda_{1}>0$, and (i) is proved.

In the same way we can show that (4.1) with $D^{\mathrm{R}} f$ instead of $f\left(D^{\mathrm{R}}\right.$ being any right-invariant differential operator on $N$ ) converges uniformly; then also (ii) is proved.

It is easy to verify that, if $1<p \leq \infty$ and $\operatorname{Im} \lambda>-Q(1-1 / p) / 2$, then $P_{a}^{1 / 2-i \lambda / Q}$ is in $L^{p}(N)$ for every $a$ in $A$.

Set $f_{a}(n)=f(n a)$ for every $n a$ in $N A$, let $1<p<\infty$ and $\operatorname{Im} \lambda \geq 0$; then by Lemma 4.2 and formula (3.1), we obtain

$$
\begin{aligned}
\|\widehat{f}(\lambda, \cdot)\|_{L^{p}(N)} & =\left\|\int_{A} f_{a} * P_{a}^{1 / 2-i \lambda / Q} a^{-Q-1} d a\right\|_{L^{p}(N)} \\
& \leq \int_{A}\left\|f_{a} * P_{a}^{1 / 2-i \lambda / Q}\right\|_{L^{p}(N)} a^{-Q-1} d a \\
& \leq \int_{A}\left\|f_{a}\right\|_{L^{1}(N)}\left\|P_{a}^{1 / 2-i \lambda / Q}\right\|_{L^{p}(N)} a^{-Q-1} d a \\
& =\int_{A} \int_{N}|f(n a)| d n a^{-Q / 2-\operatorname{Im} \lambda} a^{Q / p}\left\|P_{1}^{1 / 2-i \lambda / Q}\right\|_{L^{p}(N)} a^{-Q-1} d a \\
& \leq c\left\|P_{1}^{1 / 2}\right\|_{L^{p}(N)} \int_{e^{\tau}}^{\infty} a^{-\operatorname{Im} \lambda} a^{Q / p} a^{-Q-1} d a \\
& \leq c e^{-\tau \operatorname{Im} \lambda} .
\end{aligned}
$$

For every nonnegative integer $h$, the same holds with $\widehat{\mathcal{L}^{h} f}$ instead of $\widehat{f}$; hence

$$
\sup _{\operatorname{Im} \lambda \geq 0} e^{\tau \operatorname{Im} \lambda}\left(1+|\lambda|^{2}\right)^{h}\|\widehat{f}(\lambda, \cdot)\|_{L^{p}(N)}=\sup _{\operatorname{Im} \lambda \geq 0} e^{\tau \operatorname{Im} \lambda}\left\|\widehat{\mathcal{L}^{h} f}(\lambda, \cdot)\right\|_{L^{p}(N)}<\infty .
$$

The case $p=\infty$ can be handled in a similar way.

5. Biradial spherical analysis on $N$. Let $G$ be a connected semisimple Lie group with finite center and rank one. Fix an Iwasawa decomposition 
$G=N A K$; then $N$ is an $H$-type group [Ko2]. It is well known that $(M N, M)$ is a Gelfand pair, where $M$ is the centralizer of $A$ in $K$.

Although for nonsymmetric $N A$ spaces there is no compact group $K$ acting transitively by isometries on geodesic spheres [Da3], it is possible to obtain an analogue of the Gelfand pair $(M N, M)$ by using the notion of averaging projector given below. This will allow us to avoid the operator-valued Fourier transform (2.3) on $N$ and use a scalar-valued transform instead (see Lemma 5.4).

Let us recall some definitions first. Suppose that $S$ is a Lie group with left Haar measure $d x$. If $\varphi$ and $\psi$ are two functions in $C_{\mathrm{c}}^{\infty}(S)$, we write

$$
\langle\varphi, \psi\rangle=\int_{S} \varphi(x) \psi(x) d x
$$

and

$$
\varphi * \psi(y)=\int_{S} \varphi(y x) \psi\left(x^{-1}\right) d x, \quad \check{\varphi}(x)=\varphi\left(x^{-1}\right) \quad \forall x, y \in S .
$$

Definition [DaR1]. An averaging projector on the Lie group $S$ is a linear operator $\Pi: C_{\mathrm{c}}^{\infty}(S) \rightarrow C_{\mathrm{c}}^{\infty}(S)$ such that for every $\varphi, \psi \in C_{c}^{\infty}(S)$ the following properties hold:

(1) $\Pi^{2}=\Pi$;

(2) if $\varphi \geq 0$, then $\Pi \varphi \geq 0$;

(3) $\langle\Pi \varphi, \psi\rangle=\langle\varphi, \Pi \psi\rangle$;

(4) $\int_{S} \Pi \varphi(x) d x=\int_{S} \varphi(x) d x$;

(5) $\Pi(\varphi * \Pi \psi)=(\Pi \varphi) *(\Pi \psi)$;

(6) if $B_{\varrho}$ denotes the ball centered at the identity and of radius $\varrho$ in the given left-invariant Riemannian structure, there exists a constant $c \geq 1 \mathrm{such}$ that

$$
\operatorname{supp} \varphi \subset B_{\varrho} \Rightarrow \operatorname{supp} \Pi \varphi \subset B_{c \varrho} ;
$$

(7) $\Pi$ extends to a bounded operator from $C^{n}\left(B_{\varrho}\right)$ in $C^{n}\left(B_{c \varrho}\right)$ for every integer $n$ and every $\varrho>0$.

Note that condition (6) does not depend on the Riemannian structure chosen. In fact, it was proved in $[\mathrm{H}]$ that all left-invariant Riemannian distances are equivalent. Moreover, notice that by (3) and (7) the averaging projector $\Pi$ extends to the space of locally integrable functions on $N A$.

We say that a function $\varphi$ on $S$ is $\Pi$-radial if $\Pi \varphi=\varphi$. It was proved in [DaR1] that condition (5) can equivalently be replaced by the following two conditions:

(5a) the convolution of two $\Pi$-radial functions in $C_{\mathrm{c}}^{\infty}(S)$ is $\Pi$-radial;

(5b) if $\varphi \in C_{c}^{\infty}(S)$ is $\Pi$-radial, then so is $\check{\varphi}$. 
Now let $N$ be an $H$-type group. Damek and Ricci [DaR1, Section 3] defined an averaging projector $\Pi_{1}$ on $N$ by averaging over spheres in $\mathbf{v}$, namely

$$
\Pi_{1} \varphi(X, Z)=\int_{S_{\mathrm{v}}} \varphi(|X| \eta, Z) d s(\eta) \quad \forall(X, Z) \in N, \forall \varphi \in C_{\mathrm{c}}^{\infty}(N),
$$

where $d s(\eta)$ is the normalized surface measure on the unit sphere $S_{\mathbf{v}}$ in $\mathbf{v}$.

Here we define a slightly different operator $\Pi$, by averaging also over spheres in the center $\mathbf{z}$. Let $\varphi$ be in $C_{\mathrm{c}}^{\infty}(N)$; we define $\Pi \varphi$ by the rule

$$
\Pi \varphi(X, Z)=\int_{S_{\mathbf{v}}} \int_{S_{\mathbf{z}}} \varphi(|X| \eta,|Z| \omega) d s(\omega) d s(\eta) \quad \forall(X, Z) \in N .
$$

Hence a $\Pi$-radial function is a function that depends only on $|X|$ and $|Z|$; in accordance with $[\mathrm{Ko} 1, \mathrm{CH}]$, we will say biradial for $\Pi$-radial. Let $L^{1}(N)^{\natural}$ be the space of all biradial integrable functions on the group $N$.

Proposition 5.1. The operator $\Pi$ is an averaging projector on $N$ and $L^{1}(N)^{\natural}$ is a commutative Banach algebra.

Proof. Properties (1)-(4), (7) in the definition of averaging projector are simple to check. As for (6), recall [Ka2] that the left-invariant distance induced by the inner product on $\mathbf{n}$ between the point $(X, Z)$ and the identity $0_{N}$ depends only on $|X|$ and $|Z|$, so that (6) follows.

We will prove that condition (5) holds by checking (5a) and (5b) instead. Let $\varphi$ be a biradial function; then (5b) is immediate, for $\check{\varphi}=\varphi$. Now we check (5a), by means of the partial Radon transform in the central variable, defined as follows. For a $C_{\mathrm{c}}^{\infty}(N)$ function $\varphi$ on $N$ and a unit vector $\omega$ in $\mathbf{z}$, define the function $\mathcal{R}_{\omega} \varphi$ on $N / \exp \left(\omega^{\perp}\right)=N_{\omega}$ by the rule

$$
\mathcal{R}_{\omega} \varphi(X, \lambda)=\int_{\exp \left(\omega^{\perp}\right)} \varphi(X, \lambda \omega+Z) d Z \quad \forall X \in \mathbf{v}, \forall \lambda \in \mathbb{R} .
$$

Note that $N_{\omega}$ is isomorphic to the ordinary Heisenberg group $H^{m}$ of dimension $2 m+1$ and that the function $\varphi$ is biradial if and only if its Radon transform $\mathcal{R}_{\omega} \varphi$ does not depend on the vector $\omega$ but only on $|X|$ and $|\lambda|$.

Now take two biradial functions $\varphi$ and $\psi$ in $C_{c}^{\infty}(N)$. Since the Radon transform maps convolution on $N$ to convolution on $H^{m}$, we have

$$
\mathcal{R}_{\omega}(\varphi * \psi)=\left(\mathcal{R}_{\omega} \varphi\right) *_{H^{m}}\left(\mathcal{R}_{\omega} \psi\right) .
$$

Since $\varphi$ and $\psi$ are biradial, the right hand side of (5.1) does not depend on $\omega$. Moreover, since on the Heisenberg group $H^{m}$ convolution preserves biradial functions, we have proved that (5a) holds.

The fact that $L^{1}(N)^{\natural}$ is commutative follows easily from the commutativity of $L^{1}\left(H^{m}\right)^{\natural}$, formula (5.1), and the injectivity of the Radon transform. 
Let $\Delta_{1}$ and $\Delta_{2}$ be the left-invariant differential operators on $N$ defined by

$$
\Delta_{1}=\sum_{j=1}^{2 m} E_{j}^{2} \quad \text { and } \quad \Delta_{2}=\sum_{l=1}^{k} U_{l}^{2}
$$

where the vector fields $E_{j}$ and $U_{l}$ are defined in (2.5). Given a smooth biradial function $\varphi$ on $N$, define the function $\varphi_{0}$ on $\mathbb{R}^{+} \times \mathbb{R}^{+}$by

$$
\varphi_{0}(r, s)=\varphi(X, Z) \quad \text { with }|X|^{2}=r,|Z|^{2}=s .
$$

Straightforward computations show that the action of the operators above is given by

$$
\begin{aligned}
& \left(\Delta_{1} \varphi\right)(X, Z)=4 m \frac{d \varphi_{0}}{d r}(r, s)+4 r \frac{d^{2} \varphi_{0}}{d r^{2}}(r, s)+\frac{r}{4} \Delta_{2} \varphi(X, Z), \\
& \left(\Delta_{2} \varphi\right)(X, Z)=2 k \frac{d \varphi_{0}}{d s}(r, s)+4 s \frac{d^{2} \varphi_{0}}{d s^{2}}(r, s) .
\end{aligned}
$$

Our goal now is to find the Gelfand spectrum of the commutative algebra $L^{1}(N)^{\natural}$; as proved in [DaR1, Theorem 2.5], it consists of the bounded spherical functions, i.e., the bounded biradial eigenfunctions $\phi$ of all differential operators that commute with $\Pi$ normalized so that $\phi\left(0_{N}\right)=1$.

Lemma 5.2. Any left-invariant differential operator that commutes with the averaging projector $\Pi$ is a polynomial in $\Delta_{1}$ and $\Delta_{2}$.

Proof. By (5.3), it is clear that both $\Delta_{1}$ and $\Delta_{2}$ commute with $\Pi$. Conversely, let $D$ be a left-invariant differential operator such that $\Pi D=$ $D \Pi$. In particular, $D$ commutes with $\Pi_{1}$, so, by [DaR1, Theorem 3.3], $D$ is a polynomial in $\Delta_{1}, U_{1}, \ldots, U_{k}$ :

$$
D=\sum_{\alpha \in \mathbb{N}^{k}} P_{\alpha}\left(\Delta_{1}\right) U^{\alpha},
$$

where $P_{\alpha}$ is a polynomial and $U^{\alpha}=U_{1}^{\alpha_{1}} \ldots U_{k}^{\alpha_{k}}$ for $\alpha=\left(\alpha_{1}, \ldots, \alpha_{k}\right)$.

For any $A$ in the orthogonal group $O(k)$ over $k$ elements and any function $\varphi$ on $N$, define the function $\varphi \circ A$ on $N$ by $\varphi \circ A(X, Z)=\varphi(X, A Z)$. Suppose that $\varphi$ is a smooth biradial function on $N$; we would like to have

$$
(D \varphi)(X, A Z)=D(\varphi \circ A)(X, Z) \quad \forall(X, Z) \in N, \forall A \in O(k) .
$$

Since $\Delta_{1}$ commutes with $\Pi$, we have

$(D \varphi)(X, A Z)=\left(\sum_{\alpha} P_{\alpha}\left(\Delta_{1}\right) U^{\alpha} \varphi\right)(X, A Z)=\sum_{\alpha} P_{\alpha}\left(\Delta_{1}\right)\left(\left(U^{\alpha} \varphi\right) \circ A\right)(X, Z)$

and

$$
D(\varphi \circ A)(X, Z)=\sum_{\alpha} P_{\alpha}\left(\Delta_{1}\right) U^{\alpha}(\varphi \circ A)(X, Z) .
$$

Thus (5.4) holds if and only if $D$ is also a polynomial in $\Delta_{2}$. 
Let $\mathcal{J}_{z}$ be the function defined for every $x$ in $\mathbb{R}$ by the rule

$$
\mathcal{J}_{z}(x)= \begin{cases}\frac{\Gamma(z+1)}{\Gamma((2 z+1) / 2) \Gamma(1 / 2)} \int_{-1}^{1} e^{i x s}\left(1-s^{2}\right)^{(2 z-1) / 2} d s & \text { if } z>-1 / 2, \\ \cos x & \text { if } z=-1 / 2,\end{cases}
$$

and let $L_{d}^{\alpha}$ be the $d$ th Laguerre polynomial of order $\alpha$, i.e.,

$$
L_{d}^{\alpha}(x)=\sum_{j=0}^{d}\left(\begin{array}{l}
d+\alpha \\
d-j
\end{array}\right) \frac{(-x)^{j}}{j !} \quad \forall x \in \mathbb{R} .
$$

If $\phi$ is a spherical function, we denote by $\chi_{j}$ its eigenvalue with respect to the operator $\Delta_{j}$, i.e.

$$
\Delta_{j} \phi=\chi_{j} \phi, \quad j=1,2 .
$$

Proposition 5.3. The bounded spherical functions are

$$
\phi_{\nu, d}(X, Z)=e^{-\nu|X|^{2} / 4} \frac{L_{d}^{m-1}\left(\frac{1}{2} \nu|X|^{2}\right)}{\left(\begin{array}{c}
d+m-1 \\
d
\end{array}\right)} \mathcal{J}_{(k-2) / 2}(\nu|Z|)
$$

with eigenvalues $\chi_{1}=-\nu(2 d+m)$ and $\chi_{2}=-\nu^{2}$, and

$$
\phi_{\mu}(X, Z)=\mathcal{J}_{m-1}(\mu|X|)
$$

with $\chi_{1}=-\mu^{2}$ and $\chi_{2}=0$, where $2 m=\operatorname{dim} \mathbf{v}, k=\operatorname{dim} \mathbf{z}, \nu, \mu>0$ and integer $d \geq 0$.

Moreover, if $k \geq 2$, then $\phi_{\nu, d}$ is in $L^{q}(N)$ for every $q>2 k /(k-1)$.

Proof. By Lemma 5.2, it is enough to find the eigenfunctions of the operators $\Delta_{1}$ and $\Delta_{2}$. By (5.3) and by arguments as in [Ko1], one can check that these eigenfunctions are of the desired form.

Suppose now that $\operatorname{dim} \mathbf{z}=k \geq 2$. Then passing to polar coordinates in $\mathbf{v}$ and $\mathbf{z}$ and using well known estimates for Bessel functions (see, for example, [EMOT, vol. II, p. 85, formula (3)]), we find

$$
\begin{aligned}
\int_{N}\left|\phi_{\nu, d}(X, Z)\right|^{q} d X d Z= & c \int_{0}^{\infty} r^{2 m-1} e^{-q \nu r^{2} / 4}\left|L_{d}^{m-1}\left(\frac{1}{2} \nu r^{2}\right)\right|^{q} d r \\
& \times \int_{0}^{\infty}\left|\mathcal{J}_{(k-2) / 2}(\nu s)\right|^{q} s^{k-1} d s \\
\leq & c\left(\int_{0}^{\nu} s^{k-1} d s+\int_{\nu}^{\infty}(\nu s)^{-(k-1) q / 2} s^{k-1} d s\right) \\
\leq & c\left(\nu^{k}+\nu^{-(k-1) q / 2} \int_{\nu}^{\infty} s^{-1+k-(k-1) q / 2} d s\right),
\end{aligned}
$$

which is finite if $q>2 k /(k-1)$. 
Definition. Let $\varphi$ be a function in $L^{1}(N)^{\natural}$. We define its Gelfand transform $\mathcal{G} \varphi$ by the rule

$$
\mathcal{G} \varphi(\nu, d)=\int_{N} \varphi(n) \phi_{\nu, d}(n) d n \quad \forall \nu>0, \forall d \in \mathbb{N} .
$$

The relation between the Gelfand transform and the group Fourier transform is explained in the following lemma.

Lemma 5.4. Let $\pi_{\nu, \omega}$ be the representation of the group $N$ on the Hilbert space $\mathcal{W}_{\nu, \omega}$ defined in $(2.2)$ and $\xi$ any homogeneous polynomial in $\mathcal{W}_{\nu, \omega}$ of degree d such that $\|\xi\|_{\nu}=1$. For every function $\varphi$ in $L^{1}(N)^{\natural}$ we have

$$
\mathcal{G} \varphi(\nu, d)=\left\langle\pi_{\nu, \omega}(\varphi) \xi, \xi\right\rangle_{\nu} .
$$

Proof. Let $\psi_{\nu, \omega, d}$ be the function defined on $N$ by $\psi_{\nu, \omega, d}(n)=$ $\left\langle\pi_{\nu, \omega}(n) \xi, \xi\right\rangle_{\nu}$. One can prove $[\mathrm{CH}]$ that

$$
\left\langle d \pi_{\nu, \omega}\left(\Delta_{1}\right) \xi, \xi\right\rangle_{\nu}=-\nu(2 d+m) \quad \text { and } \quad\left\langle d \pi_{\nu, \omega}\left(\Delta_{2}\right) \xi, \xi\right\rangle_{\nu}=-\nu^{2} .
$$

Since

$$
\Delta_{j} \psi_{\nu, \omega, d}(n)=\left\langle\pi_{\nu, \omega}(n) d \pi_{\nu, \omega}\left(\Delta_{j}\right) \xi, \xi\right\rangle_{\nu}, \quad j=1,2,
$$

we deduce that $\psi_{\nu, \omega, d}$ and $\phi_{\nu, d}$ are eigenfunctions of $\Delta_{1}$ and $\Delta_{2}$ with the same eigenvalues. Since both functions attain the value 1 at the identity, we have

$$
\Pi\left(\psi_{\nu, \omega, d}\right)=\phi_{\nu, d} .
$$

Therefore, since $\Pi \varphi=\varphi$, we have

$$
\begin{aligned}
\left\langle\pi_{\nu, \omega}(\varphi) \xi, \xi\right\rangle_{\nu} & =\int_{N} \varphi(n) \psi_{\nu, \omega, d}(n) d n=\int_{N} \varphi(n) \Pi\left(\psi_{\nu, \omega, d}\right)(n) d n \\
& =\int_{N} \varphi(n) \phi_{\nu, d}(n) d n=\mathcal{G} \varphi(\nu, d) .
\end{aligned}
$$

REMARK. One could also check that the spherical functions $\phi_{\mu}$ correspond to the representations of $N$ which are trivial on the center.

TheOREM 5.5. Let $\varphi$ be a biradial function in $C_{\mathrm{c}}^{\infty}(N)$. Then the following inversion formula holds for all $n \in N$ :

$$
\varphi(n)=\frac{\left|S_{\mathbf{z}}\right|}{(2 \pi)^{Q}} \int_{0}^{\infty} \sum_{d=0}^{\infty}\left(\begin{array}{c}
d+m-1 \\
d
\end{array}\right) \mathcal{G} \varphi(\nu, d) \phi_{\nu, d}(n) \nu^{Q-1} d \nu .
$$

P r o of. From the inversion formula (2.4) for the group Fourier transform we have

$$
\varphi\left(0_{N}\right)=\frac{\left|S_{\mathbf{z}}\right|}{(2 \pi)^{Q}} \int_{0}^{\infty} \int_{S_{\mathbf{z}}} \operatorname{tr}\left(\pi_{\nu, \omega}(\varphi)\right) d s(\omega) \nu^{Q-1} d \nu .
$$

Arguing as in $[\mathrm{HR}]$, we can prove that, if $\varphi$ is biradial, then the infinitedimensional matrix $\left[\left\langle\pi_{\nu, \omega}(\varphi) \wp_{j, \nu}, \wp_{l, \nu}\right\rangle_{\nu}\right]_{j, l}$ is diagonal, where $\wp_{j, \nu}$ are the 
homogeneous polynomials defined in (2.1). By Lemma 5.4 and since the dimension of the space of homogeneous polynomials of degree $d$ is $\left(\begin{array}{c}d+m-1 \\ d\end{array}\right)$, we have

$$
\begin{aligned}
\operatorname{tr}\left(\pi_{\nu, \omega}(\varphi)\right) & =\sum_{j \in \mathbb{N}^{m}}\left\langle\pi_{\nu, \omega}(\varphi) \wp_{j, \nu}, \wp_{j, \nu}\right\rangle_{\nu} \\
& =\sum_{d=0}^{\infty} \sum_{|j|=d} \int_{N} \varphi(n)\left\langle\pi_{\nu, \omega}(n) \wp_{j, \nu}, \wp_{j, \nu}\right\rangle_{\nu} d n \\
& =\sum_{d=0}^{\infty}\left(\begin{array}{c}
d+m-1 \\
d
\end{array}\right) \mathcal{G} \varphi(\nu, d) .
\end{aligned}
$$

Therefore

$$
\varphi\left(0_{N}\right)=\frac{\left|S_{\mathbf{z}}\right|}{(2 \pi)^{Q}} \int_{0}^{\infty} \sum_{d=0}^{\infty}\left(\begin{array}{c}
d+m-1 \\
d
\end{array}\right) \mathcal{G} \varphi(\nu, d) \nu^{Q-1} d \nu .
$$

Let $\Lambda$ be the left translation, i.e., $\Lambda_{n} \varphi\left(n_{1}\right)=\varphi\left(n^{-1} n_{1}\right)$; since $\phi_{\nu, d}$ is a spherical function we have $\mathcal{G}\left(\Lambda_{n^{-1}} \varphi\right)(\nu, d)=\mathcal{G} \varphi(\nu, d) \phi_{\nu, d}\left(n^{-1}\right)$, so that

$$
\begin{aligned}
\varphi(n) & =\left(\Lambda_{n^{-1}} \varphi\right)\left(0_{N}\right) \\
& =\frac{\left|S_{\mathbf{z}}\right|}{(2 \pi)^{Q}} \int_{0}^{\infty} \sum_{d=0}^{\infty}\left(\begin{array}{c}
d+m-1 \\
d
\end{array}\right) \mathcal{G}\left(\Lambda_{n^{-1}} \varphi\right)(\nu, d) \nu^{Q-1} d \nu \\
& =\frac{\left|S_{\mathbf{z}}\right|}{(2 \pi)^{Q}} \int_{0}^{\infty} \sum_{d=0}^{\infty}\left(\begin{array}{c}
d+m-1 \\
d
\end{array}\right) \mathcal{G} \varphi(\nu, d) \phi_{\nu, d}\left(n^{-1}\right) \nu^{Q-1} d \nu \\
& =\frac{\left|S_{\mathbf{z}}\right|}{(2 \pi)^{Q}} \int_{0}^{\infty} \sum_{d=0}^{\infty}\left(\begin{array}{c}
d+m-1 \\
d
\end{array}\right) \mathcal{G} \varphi(\nu, d) \phi_{\nu, d}(n) \nu^{Q-1} d \nu,
\end{aligned}
$$

because $\phi_{\nu, d}\left(n^{-1}\right)=\phi_{\nu, d}(n)$.

One could also find the Plancherel formula by applying standard arguments.

6. The Fourier transform of the powers of the Poisson kernel. In the whole section $\nu$ denotes a positive number and $d$ a nonnegative integer. In the previous section we have proved that the spherical function $\phi_{\nu, d}$ belongs to $L^{q}(N)$ for every $q>2 k /(k-1)$. Moreover, if $\operatorname{Im} \lambda>-(Q / 2)\left(1-1 / q^{\prime}\right)$, then $P_{a}^{1 / 2-i \lambda / Q}$ is in $L^{q^{\prime}}(N)$ for every $a>0$. It follows that if $\operatorname{Im} \lambda>-(Q / 2)(1 / q)$ for some $q>2 k /(k-1)$, then, by the Hölder inequality, the function

$$
K_{\nu, d}(a, \lambda)=\int_{N} P_{a}^{1 / 2-i \lambda / Q}(n) \phi_{\nu, d}(n) d n
$$


is well defined for every $a>0$. Moreover, the function $\lambda \mapsto K_{\nu, d}(a, \lambda)$ is holomorphic in the region $\{\lambda \in \mathbb{C}: \operatorname{Im} \lambda>-(Q / 2)(1 / q)\}$.

If $\operatorname{Im} \lambda>0$, then $P_{a}^{1 / 2-i \lambda / Q}$ is in $L^{1}(N)^{\natural}$; thus, by Lemma 5.4, we have

$$
K_{\nu, d}(a, \lambda)=\left\langle\pi_{\nu, \omega}\left(P_{a}^{1 / 2-i \lambda / Q}\right) \xi, \xi\right\rangle_{\nu} \quad \text { if } \operatorname{Im} \lambda>0,
$$

for every $\omega$ in $S_{\mathbf{z}}$ and every homogeneous polynomial $\xi$ of degree $d$ such that $\|\xi\|_{\nu}=1$.

M. G. Cowling and U. Haagerup $[\mathrm{CH}]$ calculated the Fourier transform of $P_{1}^{1 / 2-i \lambda / Q}$; using their result and the identity (3.1), for $\operatorname{Im} \lambda>0$ we obtain

$$
K_{\nu, d}(a, \lambda)=a^{Q / 2-i \lambda} \alpha(\lambda) \nu^{-2 i \lambda} L\left(\nu, Q_{0} / 2-i \lambda+d, Q_{0} / 2+i \lambda+d\right),
$$

where

$$
\alpha(\lambda)=\frac{(2 \pi)^{m+1} \pi^{(k-1) / 2}}{\Gamma\left(Q_{0} / 2-i \lambda\right) \Gamma(Q / 2-i \lambda)}
$$

and, for $\nu$ in $\mathbb{R}^{+}$and $b, c$ in $\mathbb{C}$, with $\operatorname{Re} b>0$ (see [EMOT, Vol. I, p. 255]),

$$
L(\nu, b, c)=\int_{0}^{\infty} e^{-\nu(2 x+1)} x^{b-1}(x+1)^{-c} d x .
$$

By analytic continuation, (6.1) holds also if $\operatorname{Im} \lambda>-(Q / 2)(1 / q)$ for some $q>2 k /(k-1)$.

Using the equality (see [CH, Proposition 3.6])

$$
\frac{(2 \nu)^{b}}{\Gamma(b)} L(\nu, b, c)=\frac{(2 \nu)^{c}}{\Gamma(c)} L(\nu, c, b) \quad \forall b, c \in \mathbb{C},
$$

one can easily check that, if $|\operatorname{Im} \lambda|<(Q / 2)(1 / q)$ for some $q>2 k /(k-1)$, then

$$
K_{\nu, d}(a, \lambda)=\gamma_{\nu, d}(\lambda) K_{\nu, d}(a,-\lambda),
$$

where

$$
\gamma_{\nu, d}(\lambda)=(2 \nu)^{-2 i \lambda} \frac{\mathbf{c}(-\lambda) \Gamma(2 i \lambda) \Gamma\left(Q_{0} / 2-i \lambda+d\right)}{\mathbf{c}(\lambda) \Gamma(-2 i \lambda) \Gamma\left(Q_{0} / 2+i \lambda+d\right)} .
$$

Note that $\gamma_{\nu, d}$ is holomorphic in $\{\lambda \in \mathbb{C}: \operatorname{Im} \lambda<0\}$. Thus by analytic continuation (6.2) holds for every $\lambda$ in $\mathbb{C}$ and the function $\lambda \mapsto K_{\nu, d}(a, \lambda)$ is entire.

We have just proved the following

Lemma 6.1. For every positive number a, the function $K_{\nu, d}(a, \cdot)$ continues analytically to an entire function.

The factor $\gamma_{\nu, d}(\lambda)$ is essentially the Fourier transform of the convolution kernel $A_{\lambda}$ associated with the intertwining operator between the representations whose coefficients are the spherical functions as in [ADY, DoZ]. For- 
mula (6.2) is equivalent to $A_{\lambda} * P_{1}^{1 / 2-i \lambda / Q}=P_{1}^{1 / 2+i \lambda / Q}$, read on the Fourier transform side.

We now find an asymptotic expansion for $K_{\nu, d}(\cdot, \lambda)$.

LEMma 6.2. For every complex number $\lambda$ the function $K_{\nu, d}(\cdot, \lambda)$ is a solution of the differential equation

$$
\begin{aligned}
a^{2} u^{\prime \prime}(a)+(1-Q) a u^{\prime}(a)-(\nu(2 d+m) a+ & \left.\nu^{2} a^{2}\right) u(a) \\
& =-\left(\lambda^{2}+Q^{2} / 4\right) u(a) .
\end{aligned}
$$

Pr o o f. By (2.6) the Laplace-Beltrami operator $\mathcal{L}$ on $N A$ can be written in the form

$$
\mathcal{L}=a^{2} \partial_{a}^{2}+(1-Q) a \partial_{a}+a \Delta_{1}+a^{2} \Delta_{2},
$$

where $\Delta_{1}$ and $\Delta_{2}$ are the differential operators on $N$ defined by (5.2). We know by Proposition 5.3 that

$$
\Delta_{1} \phi_{\nu, d}=-\nu(2 d+m) \phi_{\nu, d} \quad \text { and } \quad \Delta_{2} \phi_{\nu, d}=-\nu^{2} \phi_{\nu, d} .
$$

Moreover, setting $\Psi_{\lambda}(n a)=P_{a}^{1 / 2-i \lambda / Q}(n)$, we have

$$
\mathcal{L} \Psi_{\lambda}=-\left(\lambda^{2}+Q^{2} / 4\right) \Psi_{\lambda} .
$$

Therefore

$$
\begin{aligned}
-\left(\lambda^{2}+Q^{2} / 4\right) K_{\nu, d}(a, \lambda) \\
=\int_{N} \mathcal{L} \Psi_{\lambda}(n a) \phi_{\nu, d}(n) d n \\
=\int_{N}\left[\left(a^{2} \partial_{a}^{2}+(1-Q) a \partial_{a} \Psi_{\lambda}\right)(n a) \phi_{\nu, d}(n)\right. \\
\left.\quad+\Psi_{\lambda}(n a)\left(\left(a \Delta_{1}+a^{2} \Delta_{2}\right) \phi_{\nu, d}\right)(n)\right] d n \\
=\left(a^{2} \partial_{a}^{2}+(1-Q) a \partial_{a}\right) K_{\nu, d}(a, \lambda)-\left(a^{2} \nu(2 d+m)+a \nu^{2}\right) K_{\nu, d}(a, \lambda) .
\end{aligned}
$$

Lemma 6.3. For $2 i \lambda$ not being a positive integer, define

$$
I_{\nu, d}(a, \lambda)=a^{Q / 2-i \lambda} \sum_{l=0}^{\infty} \beta_{l}(\lambda) a^{l}, \quad a>0,
$$

where $\beta_{-1}(\lambda)=0, \beta_{0}(\lambda)=1$, and, for $l \geq 1, \beta_{l}(\lambda)$ is given by the recursion formula

$$
l(l-2 i \lambda) \beta_{l}(\lambda)-\nu(2 d+m) \beta_{l-1}(\lambda)-\nu^{2} \beta_{l-2}(\lambda)=0 .
$$

Then the function $I_{\nu, d}(\cdot, \lambda)$ is a solution of the differential equation (6.3). Moreover, the function $\lambda \mapsto I_{\nu, d}(a, \lambda)$ is holomorphic in $\{\lambda \in \mathbb{C}: \operatorname{Im} \lambda>0\}$.

Proof. Let $v(t)=u\left(e^{t}\right)$. Then the equation (6.3) takes the form $v^{\prime \prime}-Q v^{\prime}-\left(e^{t} \nu(2 d+m)+e^{2 t} \nu^{2}\right) v=-\left(\lambda^{2}+Q^{2} / 4\right) v$. 
If $w(t)=e^{-(Q / 2-i \lambda) t} v(t)$ then

$$
w^{\prime \prime}-2 i \lambda w^{\prime}-\left(e^{t} \nu(2 d+m)+e^{2 t} \nu^{2}\right) w=0 .
$$

We try to find a solution to (6.5) of the form $\sum_{l=0}^{\infty} \beta_{l}(\lambda) e^{l t}$. Differentiating term by term in the series and substituting into (6.5) we find the recursion formula (6.4). By induction on $l$ one can prove that, if $2 i \lambda$ is not a positive integer, then there exists a constant $M_{\lambda}>0$, depending on $\lambda$, such that

$$
\left|\beta_{l}(\lambda)\right| \leq M_{\lambda}^{l} / l ! \quad \forall \lambda \geq 0 .
$$

It follows that the series $\sum_{l=0}^{\infty} \beta_{l}(\lambda) e^{l t}$ converges uniformly in $\{t \in \mathbb{R}: t \leq \tau\}$ for every real number $\tau$, so that the term by term differentiation is justified.

By induction on $l$ one can also verify that

$$
\left|\beta_{l}(\lambda)\right| \leq \sigma^{l} / l ! \text { if } \operatorname{Im} \lambda \geq 0, \forall l \geq 0,
$$

where $\sigma=\max \left(\nu(2 d+m), \nu^{2}, 1\right)$. Hence the series $\sum_{l=0}^{\infty} \beta_{l}(\lambda) a^{l}$ converges uniformly in $\{\lambda \in \mathbb{C}: \operatorname{Im} \lambda \geq 0\} \times\left\{\lambda \in \mathbb{R}: a \leq e^{\tau}\right\}$, for every real number $\tau$, and $I_{\nu, d}(a, \cdot)$ is holomorphic in the region $\{\lambda \in \mathbb{C}: \operatorname{Im} \lambda>0\}$.

Using the identity

$$
\int_{0}^{\infty}(1+t)^{-p-q} t^{p-1} d t=\frac{\Gamma(p) \Gamma(q)}{\Gamma(p+q)}
$$

one can check that

$$
\int_{N} P_{1}^{1 / 2-i \lambda / Q}(n) d n=\mathbf{c}(-\lambda) \frac{\pi^{(2 m+k+1) / 2}}{2^{k-1} \Gamma((2 m+k+1) / 2)} .
$$

For the sake of brevity write

$$
c_{m, k}=\frac{\pi^{(2 m+k+1) / 2}}{2^{k-1} \Gamma((2 m+k+1) / 2)} .
$$

LEMMA 6.4. We have

$$
K_{\nu, d}(a, \lambda)=c_{m, k}\left[\mathbf{c}(-\lambda) I_{\nu, d}(a,-\lambda)+\mathbf{c}(\lambda) \gamma_{\nu, d}(\lambda) I_{\nu, d}(a, \lambda)\right]
$$

for every $a>0$ and for every complex number $\lambda$.

Proof. If $2 i \lambda$ is not an integer then $I_{\nu, d}(\cdot,-\lambda)$ and $I_{\nu, d}(\cdot, \lambda)$ are two independent solutions of the differential equation (6.5); it follows that $K_{\nu, d}$ can be written in the form

$$
K_{\nu, d}(a, \lambda)=B_{1}(\lambda) I_{\nu, d}(a,-\lambda)+B_{2}(\lambda) I_{\nu, d}(a, \lambda) .
$$

We have to compute $B_{1}(\lambda)$ and $B_{2}(\lambda)$. By the Lebesgue dominated convergence theorem we obtain 


$$
\begin{aligned}
\lim _{a \rightarrow 0^{+}} a^{-Q / 2-i \lambda} K_{\nu, d}(a, \lambda) & =\int_{N} P_{1}^{1 / 2-i \lambda / Q}(n) \lim _{a \rightarrow 0^{+}} \phi_{\nu, d}\left(a n a^{-1}\right) d n \\
& =\int_{N} P_{1}^{1 / 2-i \lambda / Q}(n) d n \\
& =\mathbf{c}(-\lambda) \frac{\pi^{2 m+k+1}}{2^{k-1} \Gamma((2 m+k+1) / 2)}=\mathbf{c}(-\lambda) c_{m, k} .
\end{aligned}
$$

Therefore, using the identity (6.2), when $2 i \lambda$ is not an integer and $\operatorname{Im} \lambda>0$, we have

$$
B_{1}(\lambda)=c_{m, k} \mathbf{c}(-\lambda) \quad \text { and } \quad B_{2}(\lambda)=c_{m, k} \mathbf{c}(\lambda) \gamma_{\nu, d}(\lambda) .
$$

Finally, the equality of the lemma holds for every complex number $\lambda$ because $K_{\nu, d}(a, \cdot)$ is an entire function.

\section{Proof of Theorem 4.1}

Proposition 7.1. Let $f$ be in $\mathcal{S}(N A)$ and let $\widehat{f}$ be in $\mathcal{H}_{\tau, p}$ for some $p$, $1<p<2 k /(k+1)$. If $\operatorname{dim} \mathbf{z}=k>1$, then the support of $f$ is contained in $E_{\tau}$.

Proof. It is enough to prove that under the above hypotheses we have

$$
f(a)=0 \quad \text { if } a<e^{\tau} .
$$

In fact, to prove that $f(\bar{n} a)=0$ when $a<e^{\tau}$ we consider the function $\Lambda_{\bar{n}^{-1}} f(n a)=f(\bar{n} n a)$. Since $\widehat{\Lambda_{\bar{n}^{-1}}} f(\lambda, n)=\widehat{f}(\lambda, \bar{n} n)$, also $\widehat{\Lambda_{\bar{n}^{-1}} f}$ is in $\mathcal{H}_{\tau, p}$. Thus $\Lambda_{\bar{n}^{-1}} f(a)=0$ if $a<e^{\tau}$.

For $a$ in $A$, define $f_{a}$ to be the function on $N$ given by

$$
f_{a}(n)=f(n a) \quad \forall n \in N
$$

and, with a slight abuse of notation, let $\Pi f$ be the function on $N A$ given by

$$
\Pi f(n a)=\left(\Pi f_{a}\right)(n) \quad \forall n a \in N A .
$$

By the Fubini theorem, by property (5) in the definition of the averaging projector, and since $P_{a}$ is biradial, we have

$$
\begin{aligned}
\Pi \widehat{f}(\lambda, \cdot) & =\Pi\left(\int_{0}^{\infty} f_{a} * P_{a}^{1 / 2-i \lambda / Q} a^{-Q-1} d a\right) \\
& =\int_{0}^{\infty} \Pi\left(f_{a} * P_{a}^{1 / 2-i \lambda / Q}\right) a^{-Q-1} d a \\
& =\int_{0}^{\infty}\left(\Pi f_{a}\right) * P_{a}^{1 / 2-i \lambda / Q} a^{-Q-1} d a=\widehat{\Pi f}(\lambda, \cdot) .
\end{aligned}
$$


Since an averaging projector is a norm-decreasing operator on $L^{p}$ (see [DaR1, Proposition 1.3]), if $\widehat{f}$ is in $\mathcal{H}_{\tau, p}$, then also $\widehat{\Pi f}$ is in $\mathcal{H}_{\tau, p}$.

Therefore, since $f(a)=f_{a}\left(0_{N}\right)=\Pi f_{a}\left(0_{N}\right)$, to prove (7.1) we may and will suppose that $f_{a}$ is biradial. Note also that for every $\lambda$ in $\mathbb{R}$ the function $\widehat{f}(\lambda, \cdot)=\int_{0}^{\infty} f_{a} * P_{a}^{1 / 2-i \lambda / Q} a^{-Q-1} d a$ is biradial, as $f_{a}$ and $P_{a}$ are both biradial.

We will show that if $a<e^{\tau}$ then

$$
\left\langle f_{a}, \phi_{\nu, d}\right\rangle=0 \quad \forall \nu>0, d=0,1,2, \ldots
$$

Hence by Theorem 5.5 we will obtain $f_{a} \equiv 0$.

Define

$$
F_{\nu, d}(a)=\int_{N} f(n a) \phi_{\nu, d}(n) d n \quad \text { and } \quad \widehat{F}_{\nu, d}(\lambda)=\int_{N} \widehat{f}(\lambda, n) \phi_{\nu, d}(n) d n .
$$

Let $q$ be the conjugate exponent of $p$, i.e. $1 / p+1 / q=1$. Note that if $1<p<2 k /(k+1)$, then $q>2 k /(k-1)$; hence the last integral converges because of Proposition 5.3 and condition (iii) in the definition of the space $\mathcal{H}_{\tau, p}$.

If $\operatorname{Im} \lambda=0$ we get

$$
\begin{aligned}
\widehat{F}_{\nu, d}(\lambda) & =\int_{N} \int_{A} f_{a} * P_{a}^{1 / 2-i \lambda / Q}(n) a^{-Q-1} d a \phi_{\nu, d}(n) d n \\
& =\int_{A}\left\langle f_{a} * P_{a}^{1 / 2-i \lambda / Q}, \phi_{\nu, d}\right\rangle a^{-Q-1} d a \\
& =\int_{A}\left\langle f_{a}, \phi_{\nu, d}\right\rangle\left\langle P_{a}^{1 / 2-i \lambda / Q}, \phi_{\nu, d}\right\rangle a^{-Q-1} d a \\
& =\int_{A} F_{\nu, d}(a) K_{\nu, d}(a, \lambda) a^{-Q-1} d a .
\end{aligned}
$$

Thus by (6.2),

$$
\widehat{F}_{\nu, d}(\lambda)=\gamma_{\nu, d}(\lambda) \widehat{F}_{\nu, d}(-\lambda), \quad \operatorname{Im} \lambda=0 .
$$

By the inversion formula (3.2) and by Lemma 6.4 we have

$$
\begin{aligned}
F_{\nu, d}(a) & =\frac{1}{4 \pi} \int_{N} \int_{-\infty}^{\infty} \int_{N} \widehat{f}\left(\lambda, n^{\prime}\right) P_{a}^{1 / 2+i \lambda / Q}\left(n^{\prime-1} n\right) \phi_{\nu, d}(n) d n|\mathbf{c}(\lambda)|^{-2} d \lambda d n^{\prime} \\
& =\frac{1}{4 \pi} \int_{-\infty}^{\infty}\left\langle\widehat{f}(\lambda, \cdot) * P_{a}^{1 / 2+i \lambda / Q}, \phi_{\nu, d}\right\rangle|\mathbf{c}(\lambda)|^{-2} d \lambda
\end{aligned}
$$




$$
\begin{aligned}
& =\frac{1}{4 \pi} \int_{-\infty}^{\infty}\left\langle\widehat{f}(\lambda, \cdot), \phi_{\nu, d}\right\rangle\left\langle P_{a}^{1 / 2+i \lambda / Q}, \phi_{\nu, d}\right\rangle|\mathbf{c}(\lambda)|^{-2} d \lambda \\
& =\frac{1}{4 \pi} \int_{-\infty}^{\infty} \widehat{F}_{\nu, d}(\lambda) K_{\nu, d}(a,-\lambda)|\mathbf{c}(\lambda)|^{-2} d \lambda \\
& =\frac{c_{m, k}}{4 \pi} \int_{-\infty}^{\infty} \widehat{F}_{\nu, d}(\lambda)\left[\mathbf{c}(-\lambda)^{-1} I_{\nu, d}(a, \lambda)+\mathbf{c}(\lambda)^{-1} \gamma_{\nu, d}(-\lambda) I_{\nu, d}(a,-\lambda)\right] d \lambda .
\end{aligned}
$$

Therefore, by (6.2),

$$
F_{\nu, d}(a)=\frac{c_{m, k}}{2 \pi} \int_{-\infty}^{\infty} \widehat{F}_{\nu, d}(\lambda) I_{\nu, d}(a, \lambda) \mathbf{c}(-\lambda)^{-1} d \lambda .
$$

Since $\widehat{f}$ is in $\mathcal{H}_{\tau, p}$, the function $\widehat{F}_{\nu, d}$ is holomorphic in $\{\lambda \in \mathbb{C}: \operatorname{Im} \lambda>0\}$. By the Hölder inequality, if $\operatorname{Im} \lambda \geq 0$, then for every nonnegative integer $l$ there exists a constant $c$ (depending on $\nu, d$ and $l$ ) such that

$$
\begin{aligned}
\left|\widehat{F}_{\nu, d}(\lambda)\right| & \leq \int_{N}\left|\widehat{f}(\lambda, n) \phi_{\nu, d}(n)\right| d n \\
& \leq\|\widehat{f}(\lambda, \cdot)\|_{L^{p}(N)}\left\|\phi_{\nu, d}\right\|_{L^{q}(N)} \leq c\left(1+|\lambda|^{2}\right)^{-l} e^{-\tau \operatorname{Im} \lambda} .
\end{aligned}
$$

By Lemma 6.3 the function $I_{\nu, d}(a, \cdot)$ is holomorphic in $\{\lambda \in \mathbb{C}: \operatorname{Im} \lambda>0\}$; moreover, by (6.6) there exists a constant $c$ (depending on $\nu$ and $d$ ) such that

$$
\left|I_{\nu, d}(a, \lambda)\right| \leq c a^{Q / 2+\operatorname{Im} \lambda} \quad \forall a<e^{\tau}, \operatorname{Im} \lambda \geq 0 .
$$

Finally, the function $\lambda \mapsto \mathbf{c}(-\lambda)^{-1}$ is meromorphic with simple poles at $\lambda=-i(Q / 2+l), l=0,1, \ldots$, and there exists a constant $c^{\prime}$ such that

$$
\mathbf{c}(-\lambda)^{-1} \leq c^{\prime}\left(1+|\lambda|^{2}\right)^{Q} .
$$

Therefore we can shift the integration in (7.2), obtaining, for every $b>0$,

$$
F_{\nu, d}(a)=\frac{c_{m, k}}{2 \pi} \int_{-\infty}^{\infty} \widehat{F}_{\nu, d}(\lambda+i b) I_{\nu, d}(a, \lambda+i b) \mathbf{c}(-\lambda-i b)^{-1} d \lambda .
$$

Thus, by (7.3)-(7.5), we get

$$
\left|F_{\nu, d}(a)\right| \leq c a^{Q / 2+b} e^{-\tau b} \int_{-\infty}^{\infty}\left(1+\lambda^{2}+b^{2}\right)^{Q-l} d \lambda \leq c a^{Q / 2}\left(e^{-\tau} a\right)^{b}
$$

for every positive integer $l>Q+1$.

For $a<e^{\tau}$, letting $b \rightarrow \infty$, we obtain $F_{\nu, d}(a)=0$. 


\section{REFERENCES}

[ADY] J.-P. Anker, E. Damek and C. Yacoub, Spherical analysis on harmonic AN groups, Ann. Scuola Norm. Sup. Pisa 33 (1996), 643-679.

[ACD] F. Astengo, R. Camporesi and B. Di Blasio, The Helgason Fourier transform on a class of nonsymmetric harmonic spaces, Bull. Austral. Math. Soc. 55 (1997), 405-424.

[CDKR] M. Cowling, A. H. Dooley, A. Korányi and F. Ricci, An approach to symmetric spaces of rank one via groups of Heisenberg type, J. Geom. Anal., to appear.

[CH] M. G. Cowling and U. Haagerup, Completely bounded multipliers of the Fourier algebra of a simple Lie group of real rank one, Invent. Math. 96 (1989), 507-549.

[Da1] E. Damek, A Poisson kernel on Heisenberg type nilpotent groups, Colloq. Math. 53 (1987), 239-247.

[Da2] - Curvature of a semidirect extension of a Heisenberg type nilpotent group, ibid., 249-253.

[Da3] —, Geometry of a semidirect extension of a Heisenberg type nilpotent group, ibid., 255-268.

[DaR1] E. Damek and F. Ricci, Harmonic analysis on solvable extensions of $H$-type groups, J. Geom. Anal. 2 (1992), 213-248.

[DaR2] - - - A class of nonsymmetric harmonic Riemannian spaces, Bull. Amer. Math. Soc. 27 (1992), 139-142.

[Di] B. Di Blasio, Paley-Wiener type theorems on harmonic extensions of H-type groups, Monatsh. Math. 123 (1997), 21-42.

[DoZ] A. Dooley and G. Zhang, Spherical functions on H-type groups, preprint.

[EbO] P. Eberlein and B. O'N eill, Visibility manifolds, Pacific J. Math. 46 (1973), 45-109.

[EMOT] A. Erdélyi, W. Magnus, F. Oberhettinger and G. Tricomi, Higher Transcendental Functions, Vols. I, II, McGraw-Hill, New York, 1953.

[F] J. Faraut, Un théorème de Paley-Wiener pour la transformation de Fourier sur un espace riemannien symétrique de rang un, J. Funct. Anal. 49 (1982), 230-268.

[GV] R. Gangolli and V. S. Varadarajan, Harmonic Analysis of Spherical Functions on Real Reductive Groups, Ergeb. Math. Grenzgeb. 101, Springer, Berlin and New York, 1988.

[HC] Harish-Chandra, Discrete series for semisimple Lie groups, Acta Math. 116 (1966), 1-111.

[He] S. Helgason, Geometric Analysis on Symmetric Spaces, Math. Surveys Monographs 39, Amer. Math. Soc., Providence, R.I., 1994.

[H] A. Hulanicki, Subalgebra of $L_{1}(G)$ associated with laplacian on a Lie group, Colloq. Math. 31 (1974), 259-287.

[HR] A. Hulanicki and F. Ricci, A Tauberian theorem and tangential convergence for bounded harmonic functions on balls in $\mathbf{C}^{n}$, Invent. Math. 62 (1980), 325-331.

[Ka1] A. Kaplan, Fundamental solutions for a class of hypoelliptic PDE generated by composition of quadratic forms, Trans. Amer. Math. Soc. 258 (1980), 147-153.

[Ka2] -, Riemannian nilmanifolds attached to Clifford modules, Geom. Dedicata 11 (1981), 127-136. 
[Ko1] A. Korányi, Some applications of Gelfand pairs in classical analysis, in: Harmonic Analysis and Group Representations, C.I.M.E., Liguori, Napoli, 1980, 333-348.

[Ko2] -, Geometric properties of Heisenberg type groups, Adv. Math. 56 (1985), $28-38$.

[Ri1] F. Ricci, Harmonic analysis on groups of type $H$, preprint.

[Ri2] - The spherical transform on harmonic extensions of $H$-type groups, Rend. Sem. Mat. Univ. Politec. Torino 50 (1992), 381-392.

[RWW] H. S. Ruse, A. G. Walker and T. J. Willmore, Harmonic Spaces, Edizioni Cremonese, Roma, 1961.

[V] V. S. Varadarajan, Harmonic Analysis on Real Reductive Groups, Lecture Notes in Math. 576, Springer, Berlin, 1977.

Dipartimento di Matematica

Politecnico di Torino

Corso Duca degli Abruzzi, 24

10129 Torino, Italy

E-mail: astengo@calvino.polito.it diblasio@calvino.polito.it

Received 24 February 1998;

revised 16 October 1998 